\title{
GM-CSF Grown Bone Marrow Derived Cells Are Composed of Phenotypically Different Dendritic Cells and Macrophages
}

\author{
Yi Rang Na, Daun Jung, Gyo Jeong Gu, and Seung Hyeok Seok ${ }^{*}$
}

Granulocyte-macrophage colony stimulating factor (GMCSF) has a role in inducing emergency hematopoiesis upon exposure to inflammatory stimuli. Although GM-CSF generated murine bone marrow derived cells have been widely used as macrophages or dendritic cells in research, the exact characteristics of each cell population have not yet been defined. Here we discriminated GM-CSF grown bone marrow derived macrophages (GM-BMMs) from dendritic cells (GM-BMDCs) in several criteria. After C57BL/6J mice bone marrow cell culture for 7 days with GM-CSF supplementation, two main populations were observed in the attached cells based on MHCII and F4/80 marker expressions. GM-BMMs had $\mathrm{MHClI}^{\text {low }} \mathrm{F} 4 / 80^{\text {high }}$ as well as

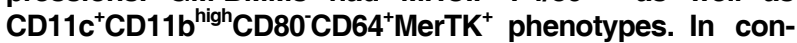
trast, GM-BMDCs had MHCII ${ }^{\text {high }} \mathrm{F} 4 / 80^{\text {low }}$ and CD11 ${ }^{\text {high }} \mathrm{CD} 8 \alpha^{-}$

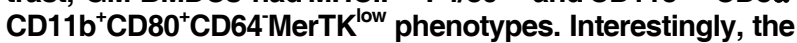
GM-BMM population increased but GM-BMDCs decreased in a GM-CSF dose-dependent manner. Functionally, GMBMMs showed extremely high phagocytic abilities and produced higher IL-10 upon LPS stimulation. GM-BMDCs, however, could not phagocytose as well, but were efficient at producing TNF $\alpha$, IL-1 $\beta$, IL-12p70 and IL- 6 as well as inducing $\mathbf{T}$ cell proliferation. Finally, whole transcriptome analysis revealed that GM-BMMs and GM-BMDCs are overlap with in vivo resident macrophages and dendritic cells, respectively. Taken together, our study shows the heterogeneicity of GM-CSF derived cell populations, and specifically characterizes GM-CSF derived macrophages compared to dendritic cells.

\section{INTRODUCTION}

M Granulocyte-macrophage colony stimulating factor (GM-CSF) mediates various signals for cellular proliferation and survival,

Department of Microbiology and Immunology, and Institute of Endemic Disease, Seoul National University College of Medicine, Seoul 03080, Korea

*Correspondence: lamseok@snu.ac.kr

Received 28 June, 2016; revised 18 September, 2016; accepted 19 September, 2016; published online 28 October, 2016

Keywords: dendritic cell, GM-CSF, macrophage, phenotype especially in hematopoietic cells (Hercus et al., 2009). As one can easily suppose from its name, GM-CSF helps form hematopoietic progenitor colonies, which can differentiate into granulocytes or macrophages. Although serum GM-CSF levels are distinctively absent in the steady state, (Cebon et al., 1994; Cheers et al., 1988) production rapidly increases upon exposure to inflammatory stimuli. Therefore GM-CSF has a role in inducing emergency hematopoiesis and not in the steady state. In this way, it is not surprising that mice with GM-CSF or GMCSF receptor deficiency showed almost normal hematopoietic cell composition. Only minor cell populations were affected by GM-CSF deficiency. Alveolar macrophages showed reduced TNF- $\alpha$ and leukotrienes, indicating that GM-CSF has tissue specific activity in alveolar macrophages (Paine et al., 2001). In addition, elicited peritoneal macrophages (Becker et al., 2012) as well as inflammatory dendritic cells (DCs) were also diminished in GM-CSF deficient mice suggesting that GM-CSF has a role in inflammation.

For mice research, the relative numbers of macrophages to be obtained from mice are low so isolating sufficient numbers for comprehensive studies can be logistically burdensome. Therefore the majority of applications currently rely on the in vitro generation of macrophages from murine bone marrow (BM) cells with the appropriate haematopoietic growth factor MCSF (Xu et al., 2007). M-CSF grown BM-derived macrophages (M-BMMs) are used commonly because of their resemblance to resident homeostatic macrophages, and M-CSF can produce relatively homogenous macrophage populations in vitro. In contrast, although GM-CSF grown BM-derived macrophages (GM-BMM) are also accepted as source of macrophages, (Murray et al., 2014) they are contaminated with other cell types, predominantly DCs. Nonetheless, GM-BMMs still have some value as a macrophage source (Fleetwood et al., 2007) because GM-BMMs have different functional and phenotypic characteristics from M-BMMs that are similar to inflammatory macrophages such as thioglycollate-induced peritoneal macrophages (Becker et al., 2012; Fleetwood et al., 2007). Thus there are some benefits to use GM-BMMs such as in screening anti-inflammatory drugs or investigating the biology of inflammatory macrophages in inflamed tissues, for example. However, general experimental protocols produce GM-CSF grown BM-derived dendritic cells (GM-GMDCs) as well as GM-BMMs. Thus, we need to clarify the exact characteristics and composition of each population.

In this study, we compared GM-BMMs and GM-BMDCs pro- 
duced in the same culture environment and found several differences. We found differences in cell surface markers, profiles of cytokine secretion, cell morphology, ability to phagocytize latex beads and ability to proliferate T cells. We also found that microarray profiling of GM-BMMs overlaps with resident macrophages, and GM-BMDCs overlaps with resident DCs in vivo. Thus, we present here the heterogeneity of GM-CSF grown bone marrow cells, and the functional and phenotypic characteristics of GM-BMMs and GM-BMDCs.

\section{MATERIALS AND METHODS}

\section{Cell preparation}

C57BL/6J mice were obtained from Jackson Laboratory. Male mice of five to ten weeks of age were used to isolate bone marrow cells. The mice were housed under specific pathogen-free (SPF) conditions and cared according to the Guide for the Care and Use of Laboratory Animals prepared by the Institution of Animal Care and Use Committee (IACUC) of Seoul National University. All of the experiments were approved by the IACUC of the Seoul National University (accession number SNU-130311-2-2). Isolated bone marrow cells from femurs and tibiae were cultured for 7 days at a density of $10^{6} / \mathrm{ml}$ in RPMI medium (Thermo, USA) supplemented with 10\% FBS (Gibco, Carlsbad, California, USA), 10 units $/ \mathrm{ml}$ penicillin, $10 \mu \mathrm{g} / \mathrm{ml}$ streptomycin, $2 \mathrm{mM} \mathrm{L-}$ glutamine (Gibco, USA) (hereafter termed complete medium) and $25 \mathrm{ng} / \mathrm{ml}$ murine GM-CSF (Miltenyi Biotech, Germany) at $37^{\circ} \mathrm{C}$ in a humidified atmosphere with $5 \% \mathrm{CO}_{2}$. On day 3 , floating cells were discarded and fresh medium containing 25 $\mathrm{ng} / \mathrm{ml}$ GM-CSF was added. Cells were further differentiated for 4 days with GM-CSF containing complete medium. Floating and attached cells were separately examined for their surface marker expressions and we obtained attached cells in this study by scrapping after gently washing the culture plates with warm PBS twice.

\section{Flow cytometry}

Floating cells and attached cells were separately prepared for FACS analysis. Cells were then incubated for $20 \mathrm{~min}$ with antibodies diluted at the optimal concentrations in FACS buffer (PBS, 5\% FBS, $5 \mathrm{mM}$ EDTA, and 1\% $\mathrm{NaN}_{3}$ ). LSRII (BD) was used for multiparameter analysis of stained cell suspensions, followed by analysis with FlowJo software (Tree Star) as described previously (Seok et al., 2013). Monoclonal antibodies to mouse F4/80 (BM8), CD11b (M1/70), IA/IE (M5/114.15.2), CD8 $\alpha$ (53-6.7), CD115 (AFS98), FceRI (MAR-1), Siglec F (E50-2440, BD Biosciences), Ly6C (HK1.4), MerTK (clone 125518, R\&D systems), CD64 (X54-5/7.1, BD Biosciences), CD11c (HL3), CD80 (16-10A1, BD Biosciences), CD86 (GL1), and CD103 (2E7), were all from eBioscience, unless indicated. $\mathrm{MHCII}{ }^{\text {high }} \mathrm{F} 4 / 80^{\text {low }}$ and MHCII ${ }^{\text {low }} \mathrm{F} 4 / 80^{\text {high }}$ populations were sorted with a FACSAria II (BD). Sorting purity was always checked and confirmed that was up to $95 \sim 98 \%$.

\section{Latex bead phagocytosis}

Bone marrow cells were differentiated in a 12-well plate at a density of $10^{6} / \mathrm{ml}$ for 7 days. After media change, cells were incubated with $1 \mu \mathrm{m}$ diameter Alexa 350-tagged latex beads (Molecular Probe) for $2 \mathrm{~h}$ at $10^{7}$ concentration per each well. Phagocytozed beads counts were analyzed on a LSRII flow cytometer (BD Biosciences) and analyzed using FlowJo software. For imaging of beads phagocytozed by DCs and macrophages, differentiated cells were sorted into DCs
( $\mathrm{MHCl}{ }^{\text {high }} \mathrm{F} 4 / 80^{\text {low }}$ population) and macrophages ( $\mathrm{MHClI}{ }^{\text {low }} \mathrm{F} 4 /$ $80^{\text {high }}$ population) and seeded in a 4-chamber well slide. After beads incubation for $2 \mathrm{~h}$, attached cells were washed three times, fixed with $4 \%$ paraformaldehyde for $10 \mathrm{~min}$, mounted and photographed using a Leica AF6000 fluorescence microscope.

\section{Cytokine production}

Sorted DCs and macrophages were seeded in 96-well plates at $5 \times 10^{4} / 200 \mu \mathrm{l}$ with complete media and rested for $6 \mathrm{~h}$. Cells were stimulated with LPS $(100 \mathrm{ng} / \mathrm{ml})$ or Polyl:C $(50 \mu \mathrm{g} / \mathrm{ml})$ for $24 \mathrm{~h}$. Supernatants were collected and stored at $-80^{\circ} \mathrm{C}$ until TNF $\alpha$, IL-12p70, IL-6 and IL-10 quantification. For IL-1 $\beta$ quantitation, cells at $24 \mathrm{~h}$ time point were lysed in RIPA buffer and stored at $-80^{\circ} \mathrm{C}$. Cells in each well were analyzed for their protein concentration using the BCA assay kit (Thermo, USA) as a normalization factor for cytokine quantitation. Cytokine concentrations were determined using the duoset ELISA kit (R\&D systems, USA).

\section{Mixed leukocyte reaction}

DCs and macrophages were sorted according to the above description. For M-CSF grown macrophages, C57BL/6J bone marrow cells were differentiated with $20 \%$ L929 culture supernatant for 7 days and sorted for the $\mathrm{F} 4 / 80^{+} \mathrm{CD} 11 \mathrm{~b}^{+}$population. To prepare $\mathrm{T}$ cells, Balb/c splenocytes were isolated and incubated in a $100 \pi$ dish for $2 \mathrm{~h}$ to remove attached cells. Floating splenocytes were collected and labeled with $5 \mu \mathrm{M}$ CFSE (Molecular Probe) for $5 \mathrm{~min}$ at $37^{\circ} \mathrm{C}$. Cells were washed with complete medium twice and seeded in a 96 -well $U$ bottom plate for mixed leukocyte reaction. Total $2 \times 10^{4}$ of antigen presenting cells and $10^{5}$ of splenocytes were mixed and incubated for 5 days. $\mathrm{CD}^{+} \mathrm{T}$ cell proliferation was examined using FACS analysis.

Microarray analysis, normalization and data analysis. RNA was prepared from sorted DCs and macrophages with Trizol reagent (Invitrogen, USA) according to the manufacturer's instructions. Global gene expression analysis was performed using Affymetrix GeneChip ${ }^{\circledR}$ Mouse Gene 1.0 ST oligonucleotide arrays. Sample preparation was performed according to the instructions and recommendations provided by the manufacturer. Expression data were generated by Affymetrix Expression Console software version1.1. For normalization, the RMA (Robust Multi-Average) algorithm was implemented in Affymetrix Expression Console software. Obtained microarray data of GM-BMDCs, GM-BMMs and MBMMs in this study was assigned a GEO accession number (GSE65425). Microarray gene expression data for lung DC, mesenteric lymph node CD8 ${ }^{+} \mathrm{DC}$, splenic CD $11 \mathrm{~b}^{+} \mathrm{DC}$, thioglycollate induced $\mathrm{F} 4 / 80^{\text {high }}$ macrophage, lung $\mathrm{CD} 11 \mathrm{~b}^{+}$macrophage and microglia were collected from the Immunological Genome Project (GSE15907) (Gautier et al., 2012; Heng et al., 2008). Expression data are pooled from 2 replicate microarray experiments.

For real-time PCR, cDNA was synthesized from 1- $\mathrm{gg}$ total RNA using M-MLT reverse transcriptase (Enzynomics, Korea). Reverse-transcribed RNA (20 ng) was used as template for quantitative real-time PCR and set up in 96-well plates using Taqman Universal Mastermix II (Applied Biosystems, USA). The primers for RelB detection were: 5d-GTTCCAGTGA CCTCTCTTCCC-3' (Forward), 5'-CCAAAGCCGTTCTCCTT AATGTA-3' (Reverse); for MafB detection were: 5'-GTTATA GGGGAGGTCTAGGTGT-3TAT (Forward), 5'-AAGCTCGTTT 
A Attaching cells GM-BMDC GM-BMM

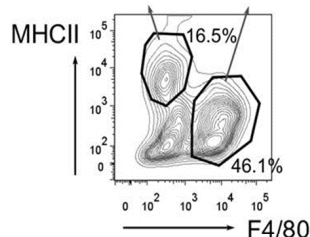

Floating cells

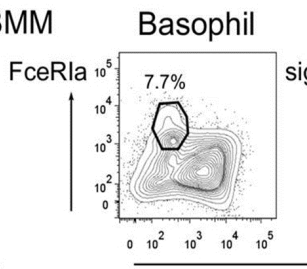

Eosinophil

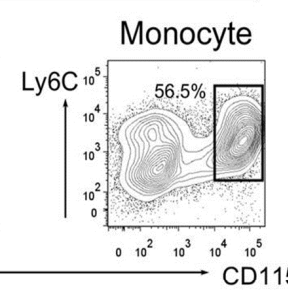

B

\begin{tabular}{|c|c|c|c|}
\hline Population & Percentage (\%) & State & Marker \\
\hline GM-BMDC & $18.3 \pm 4.6$ & Attaching & $\mathrm{MHCII}^{\text {high }} \mathrm{F} 4 / 80^{\text {low }}$ \\
\hline GM-BMM & $56.9 \pm 11.4$ & Attaching & MHCII ${ }^{\text {low }} \mathrm{F} 4 / 80^{\text {high }}$ \\
\hline Basophil & $4.6 \pm 3$ & Floating & CD115 ${ }^{-} \mathrm{Fc} \varepsilon \mathrm{RI}^{+}$ \\
\hline Eosinophil & $3.6 \pm 1.1$ & Floating & $\mathrm{CD} 115$ SiglecF $^{+}$ \\
\hline Monocyte & $56.5 \pm 1.6$ & Floating & $\mathrm{CD}_{115^{+}} \mathrm{CD} 11 \mathrm{~b}^{+}$ \\
\hline
\end{tabular}

Fig. 1. Population composition of GMCSF derived bone marrow cells. (A) Bone marrow cells were isolated from C57BL/6J mice and cultured with 25 $\mathrm{ng} / \mathrm{ml}$ for 7 days. On day 3 , fresh medium containing GM-CSF was added. Attached and floating cells were examined for the indicated marker expressions using flow cytometry. Contour plots are representative of five independent experiments. (B) A table describing the population percentages and phenotypes of GM-BMDCs, GMBMMs, basophils, eosinophils and monocytes in GM-CSF derived bone marrow cells. Percentage indicates mean \pm SEM. $n=3$

\section{A GM-BMDC GM-BMM}
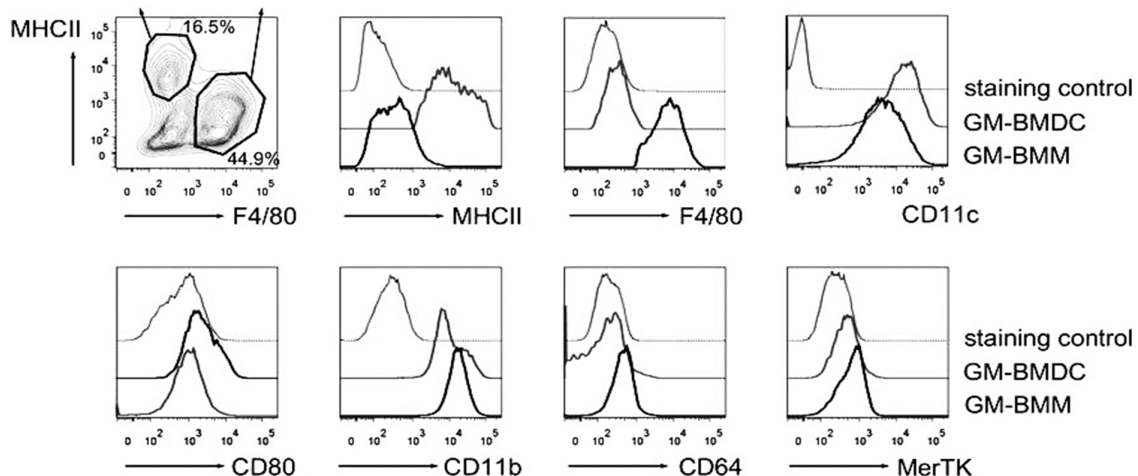

GM-BMM
GM-BMDC
Fig. 2. Surface marker expressions of GM-BMDCs and GM-BMMs. (A) GMBMDCs and GM-BMMs were gated as $\mathrm{MHCII}^{\text {high }} \mathrm{F} 4 / 80^{\text {low }}$ and $\mathrm{MHCII}{ }^{\text {low }} \mathrm{F} 4 / 80^{\text {high }}$ populations respectively, and further analyzed for the indicated marker expressions using flow cytometry. Histograms are representative of three independent experiments. (B) A table describing mean fluorescence intensity (mean \pm SEM, $\mathrm{n}=3$ ) of examined surface markers analyzed by flow cytometry.

B

\begin{tabular}{c|c|c}
\hline Marker & GM-BMDC & GM-BMM \\
\hline MHCII & $22,865 \pm 12011$ & $361 \pm 150$ \\
\hline F4/80 & $331 \pm 48$ & $7,458 \pm 4,087$ \\
\hline CD11c & $20,300 \pm 436$ & $7,394 \pm 102$ \\
\hline CD80 & $3,148 \pm 36$ & - \\
\hline CD11b & $16967 \pm 2,603$ & $18933 \pm 289$ \\
\hline CD64 & - & $458 \pm 47$ \\
\hline MerTK & $549 \pm 95$ & $628 \pm 75$
\end{tabular}

CCGATGCAG-3' (Reverse), for Maf detection were: 5'-CTGC CGCTTCAAGAGGGTGCAG C-3' (Forward), 5'-GATCTCC TGCTTGAGGTGGTC-3' (Reverse). Gene expression levels were quantified using an $\mathrm{ABI}$ Prism 7900 sequence detection system (Applied Biosystems). The relative expression of each sample was normalized to 18Sr-RNA (Applied Biosystems) and compared with the controls according to the relative $\mathrm{Ct}$ method.

Statistical analysis

All data unless otherwise indicated are shown as mean \pm SEM and were tested using two-tailed Student's $t$ test or two-way ANOVA using GraphPad Prism 4.

\section{RESULTS}

GM-CSF generates various cell populations from bone marrow cells

As GM-CSF is known to induce the expansion of various myeloid lineages, we cultured bone marrow cells in the presence of GM-CSF for 7 days to examine the exact cell populations derived from GM-CSF in vitro culture. We found that attached cells were mainly composed of two populations, based on the $\mathrm{MHCII}$ and $\mathrm{F} 4 / 80$ expressions. We assumed that $\mathrm{MHCII}^{\text {high }}$ $\mathrm{F} 4 / 80^{\text {low }}$ and $\mathrm{MHCIl}{ }^{\text {low }} \mathrm{F} 4 / 80^{\text {high }}$ populations correspond to DCs and macrophages, respectively. These GM-CSF grown, bone marrow cell derived DCs (GM-BMDCs) comprised up to $18 \%$ of 
A

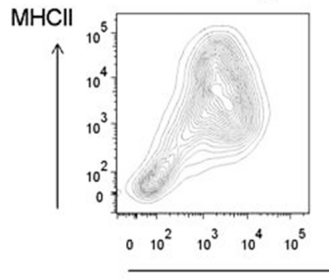

B

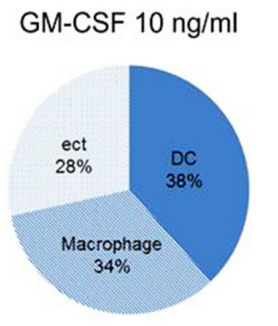

GM-CSF $10 \mathrm{ng} / \mathrm{m}^{\prime}$

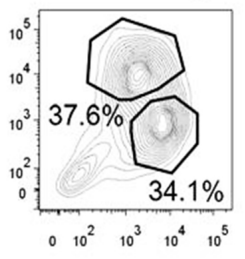

GM-CSF $25 \mathrm{ng} / \mathrm{ml}$

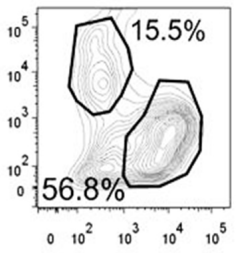

GM-CSF $100 \mathrm{ng} / \mathrm{ml}$

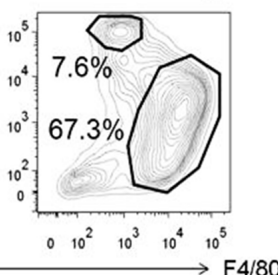

Fig. 3. Increasing GM-CSF concentration favors GM-BMMs development. (A) Dot plots of $\mathrm{MHCll}$ and $F 4 / 80$ expressions on cultured bone marrow cells with varying concentrations of GM-CSF. Bone marrow cells were differentiated with $5,10,25$ or $100 \mathrm{ng} / \mathrm{ml}$ of GM-CSF for 7 days. Attached cells were examined for their population compositions of GMBMDCs and GM-BMMs. Data are representative of three independent experiments. (B) Quantitative graph showing relative population compositions of GM-BMDCs and GM-BMMs examined in $(A)$.

total attached cells. In contrast, GM-CSF grown bone marrow cell derived macrophages (GM-BMMs) were the main cells ( $57 \%$ of the attaching cells) of the mixed populations. Floating cells consisted of monocytes (CD115 $\left.{ }^{+} \mathrm{CD} 11 \mathrm{~b}^{+}, 56.5 \pm 1.6 \%\right)$, basophils (CD115 FcERl $\alpha^{+}, 4.6 \pm 3 \%$ ) and eosinophils (CD115 SiglecF $F^{+}, 3.6 \pm 1.1 \%$ ) and we did not observe neutrophils $\left(\mathrm{Gr}^{+} \mathrm{CD} 11 \mathrm{~b}^{+} \mathrm{F} 4 / 80^{-}\right)$(Fig. 1B). All of these cell populations were $\mathrm{CD}_{11 \mathrm{~b}^{+}}$(data not shown). Much of the floating cells expressed $\mathrm{F} 4 / 80$ and they seemed to be transitioning from monocytes to macrophages or DCs. Monocyte expression of Ly6C was heterogenous and up to $54 \%$ of monocytes were Ly6C ${ }^{+}$(Fig. 1A). From these results, we revealed that attached cells isolated from GM-CSF grown bone marrow cell cultures were heterogenous and were relatively favored into differentiation of $\mathrm{MHCll}{ }^{\text {low }} \mathrm{F} 4 / 80^{\text {high }}$ macrophages.

\section{GM-CSF derived DCs and macrophages have distinct surface marker expressions}

DCs and macrophages themselves show heterogenous phenotypes in vivo (Hashimoto et al., 2011). To characterize GMBMDCs and GM-BMMs more clearly, we next investigated the surface marker expressions of these cells in detail (Fig. 2). As expected, MHCI ${ }^{\text {high }} \mathrm{F} 4 / 80^{\text {low }} \mathrm{GM}-\mathrm{BMDCs}$ expressed high levels of CD11c and CD11b. GM-BMMs expressed relatively higher levels of CD11b but lower levels of CD11c compared to GMBMDCs (Fig. 2A). GM-BMMs also expressed more CD64 and MerTK, which are known resident macrophage markers (Gautier et al., 2012). CD80 expression was only observed on GM-BMDCs. Taken together, we show that GM-CSF differentiates mixed DC and macrophage populations with distinct marker expressions in that GM-BMDC has $\mathrm{MHCI}^{\text {high }} \mathrm{F} 4 /$ $80^{\text {low }} \mathrm{CD} 11 \mathrm{c}^{\text {high }} \mathrm{CD} 8 \alpha^{-} \mathrm{CD} 11 \mathrm{~b}^{+} \mathrm{CD} 80^{+} \mathrm{CD} 64^{-} \mathrm{MerTK}^{\text {low }}$ phenotype and $\mathrm{GM}-\mathrm{BMM}$ has $\mathrm{MHCll}{ }^{\text {low }} \mathrm{F} 4 / 80^{\text {high }} \mathrm{CD} 11 \mathrm{c}^{+} \mathrm{CD} 11 \mathrm{~b}^{\text {low }} \mathrm{CD} 80^{-}$ $\mathrm{CD}^{+} 4^{+} \mathrm{MerTK}^{+}$phenotype.

\section{Macrophage population is increased dependent of} GM-CSF concentration

GM-CSF concentration varies depending on inflammatory conditions in vivo. Because GM-CSF can induce different signaling pathways depending on the extracellular concentration (Hercus et al., 2009), we investigated dose dependent effects of GM-CSF on the ratio of DCs and macrophages differentiation in in vitro culture conditions. We treated bone marrow cells with $5,10,25$ and $100 \mathrm{ng} / \mathrm{ml}$ of GM-CSF and cultured them for 7 days. At $5 \mathrm{ng} / \mathrm{ml}$, we could not distinguish DC and macrophage populations clearly in a $\mathrm{MHCll}$ and F4/80 scatter plot (Fig. 3A). Two populations were distinguished with treatment of $10 \mathrm{ng} / \mathrm{ml}$ of GM-CSF. At the usual concentration $(25 \mathrm{ng} / \mathrm{ml})$ of GM-CSF, the DC:macrophage ratio increased to 1:3 1:4 as already shown in Figs. 1 and 2. Interestingly, GM-BMDCs decreased in a GM-CSF dosedependent manner. At $100 \mathrm{ng} / \mathrm{ml}, 67.3 \%$ of attached cells were GM-BMMs but only $7.6 \%$ was comprised of GM-BMDCs. A quantitative population graph of GM-BMDCs and GMBMMs with various doses of GM-CSF is depicted in Fig. 3B. From this results, we can see that high dose of GM-CSF favors macrophage differentiation.

\section{GM-BMMs have enhanced phagocytic ability compared to GM-BMDCs}

Macrophages are efficient at phagocytosis and scavenging cellular debris in vivo. To compare the functional differences between GM-BMDCs and GM-BMMs, we first examined their phagocytic abilities. As expected, only $7.7 \%$ of $\mathrm{MHCII}^{\text {high }} \mathrm{F} 4$ $180^{\text {low }} \mathrm{GM}-\mathrm{BMDCs}$ contained beads. In contrast, most $\mathrm{MHCII}^{-}$ ${ }^{\mathrm{ow}} \mathrm{F} 4 / 80^{\text {high }} \mathrm{GM}-\mathrm{BMM}$ ( $\left.76.8 \%\right)$ had beads, illustrating their superior phagocytic ability (Fig. 4A). Bead count per cell was also significantly different between GM-BMDCs and GM-BMMs (Fig. $4 \mathrm{~B}$ ). The $7.3 \%$ of DCs had an uptake of 1 to 2 beads per cell, whereas $27.2 \%$ of macrophages had an uptake of more than 5 beads per cell. In accordance with this data, we obtained enriched phagocytosis gene ontology biological processes (GO: $\mathrm{BP}$ ) in GM-BMMs by microarray analysis (Table 1). To observe any cellular morphology differences between the two populations, we seeded sorted GM-BMDCs and GM-BMMs in a chamber well slide, incubated them with latex beads and imaged them using fluorescence microscope (Fig. 4C). GMBMDCs were relatively round and had many dendrites. On the other hand, GM-BMMs seemed to be flatter, had no evident dendrites and had many beads in their cytoplasm. Collectively, these results demonstrated that GM-CSF derived macrophages have sufficient phagocytic ability but DCs do not. 
A
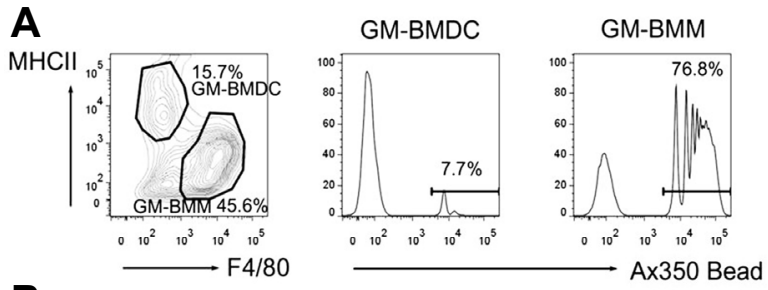

B

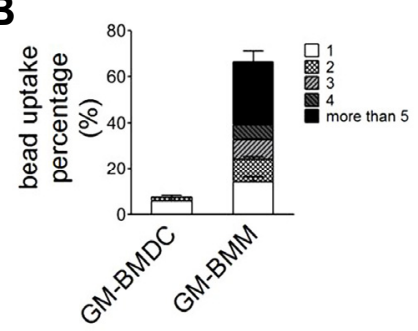

C

GM-BMDC
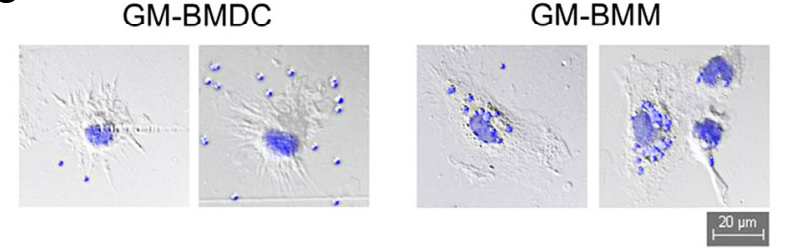

Fig. 4. Bead phagocytosis by GM-BMDCs and GM-BMMs. (A) Gated GM-BMDCs and GM-BMMs by MHCII and F4/80 expressions (left dot plot) were further analyzed for bead uptake. Alexa 350-tagged latex beads were incubated with differentiated GM-CSF derived bone marrow cells for $2 \mathrm{~h}$, washed twice and labeled with IA/IE and F4/80 antibodies. Internalized beads were analyzed using flow cytometry (Middle and right histograms). Data are representative of three independent experiments. (B) Quantitative bar graph indicating the cell percentages with internalized bead counts of GMBMDCs and GM-BMMs. Error bars, SEM. $n=3$ (C) Cell morphologies of sorted GM-BMDCs and GM-BMMs after bead internalization were photographed using fluorescence microscope. Beads are shown as blue with $1 \mu \mathrm{m}$ diameter inside or around the cells.

\section{GM-BMDCs produce much more pro-inflammatory cytokines compared to GM-BMMs}

Both DCs and macrophages are known to produce inflammatory cytokines and stimulate innate immune responses upon Tolllike receptor ligation. To study the cytokine responses of GMBMDCs and GM-BMMs, we sorted the two populations and stimulated them with Myd88/TRIF dependent TLR4 agonist LPS or TRIF dependent TLR3 agonist Polyl:C for $24 \mathrm{~h}$ (Fig. 5). Interestingly, GM-BMDCs synthesized much more TNF $\alpha$, IL$12 p 70$ and IL-6 compared to GM-BMMs upon LPS stimulation. Pro-IL-1 $\beta$ synthesis was not significantly different between DCs and macrophages. In contrast, anti-inflammatory cytokine IL-10 was produced two times higher in GM-BMMs than GM-BMDCs. LPS induced comparable effects on cytokine production, however Polyl:C did not, demonstrating that the effects of the Myd88 dependent pathway might be different in GM-CSF derived DCs and macrophages. IL-10 synthesis depends on endogenous ERK contents and it is known that macrophages are the main producers of IL-10 in an ERK-dependent manner, (Saraiva and O'Garra, 2010) further supporting our results. Taken together, we confirmed that GM-BMDCs have the ability to produce pro-inflammatory cytokines TNF $\alpha$, IL-12p70 and IL-
6 than GM-BMMs upon LPS stimulation. Conversely, GM-BMMs produce much more IL-10 than DCs.

GM-BMDCs have enhanced T-cell proliferation ability than GM-BMIMs

DCs are professional antigen presenting cells with an ability to stimulate T cells (Banchereau and Steinman, 1998). To ascertain $\mathrm{T}$ cell proliferating ability of GM-BMDCs, we performed mixed leukocyte reaction (Fig. 6). CD11 $\mathrm{b}^{+} \mathrm{F} 4 / 80^{+} \mathrm{M}-\mathrm{CSF}$ grown bone marrow derived macrophages (M-BMMs) were also examined to compare $\mathrm{T}$ cell proliferation ability. Sorted GMBMDCs or GM-BMMs were mixed with allogeneic MHCIIcarrying Balb/c slpenocytes for 5 days and CFSE-labeled CD4 ${ }^{+}$ $T$ cells were examined for their proliferation by flow cytometry. As expected, GM-BMDCs expanded T cells most extensively, resulting in the proliferation of $90.1 \%$ of $C D 4^{+} \mathrm{T}$ cells (Fig. 6A). GM-BMMs and M-BMMs resulted in $65.3 \%$ and $40.9 \%$ of proliferated $\mathrm{T}$ cells, respectively. In support of this, we also obtained enriched GO: BP of antigen processing and peptide presentation or polysaccharide antigen presentation via $\mathrm{MHC}$ class II in DC population by microarray analysis (Table 2). A quantitative graph showing proliferated $\mathrm{CD} 4^{+} \mathrm{T}$ cells reveals sequential $T$ cell stimulation abilities of GM-BMDCs, GM-BMMs and M-BMMs (Fig. 6B).

Whole transcriptome analysis shows that GM-BMDCs and GM-BMMs are clustered with tissue resident DCs and macrophages, respectively

Due to the ambiguity of characterizing cell populations using surface markers or specific functions, we sought to define GMBMDCs and GM-BMMs based on their profiles of gene transcripts (Satpathy et al., 2012). Using gene hierarchical clustering mapping and principal component analysis (PCA) of tissue resident DC and macrophage populations from the ImmGen Project (Gautier et al., 2012), we confirmed that in vitrogenerated GM-BMDCs and GM-BMMs resemble organ DCs and macrophages, respectively. Surprisingly, GM-BMMs overlap with M-BMMs as well as CD11 $\mathrm{b}^{+}$lung macrophages, microglia and thioglycollate induced peritoneal macrophages, but not with GM-BMDCs (Fig. 7A). As shown in the PCA result of all expressed genes (Fig. 7B), although resident macrophages from three specific organs have diverse gene expression patterns compared to DC populations from mesenteric lymph node, lung and spleen, GM-BMDCs most resemble tissue resident DC populations, and GM-BMMs as well as M-BMMs most resemble resident macrophage populations when comparing 2 principle component criteria. Finally, we compared lineage specific transcription factor gene expressions by real-time PCR. Expression of RelB, a transcription factor of DC (Egawa et al., 2013), was significantly higher in sorted GM-BMDC than GMBMMs (Fig. 7C). In contrast, gene expression levels of MafB and Maf, both of which are used as macrophage lineage specific markers, were higher in sorted GM-BMMs than GM-BMDCs (Fig. 7D). Collectively, our results indicated that GM-CSF generates mixed DC and macrophage populations in in vitro culture conditions, and these share some degree of similarities to their corresponding in vivo populations.

\section{DISCUSSION}

GM-CSF grown bone marrow cells are widely used as a model system for DC development and function (Nikolic et al., 2003; Zhang et al., 1998), although bioinformatics analysis of their transcriptome indicates that they are closer to macrophages 

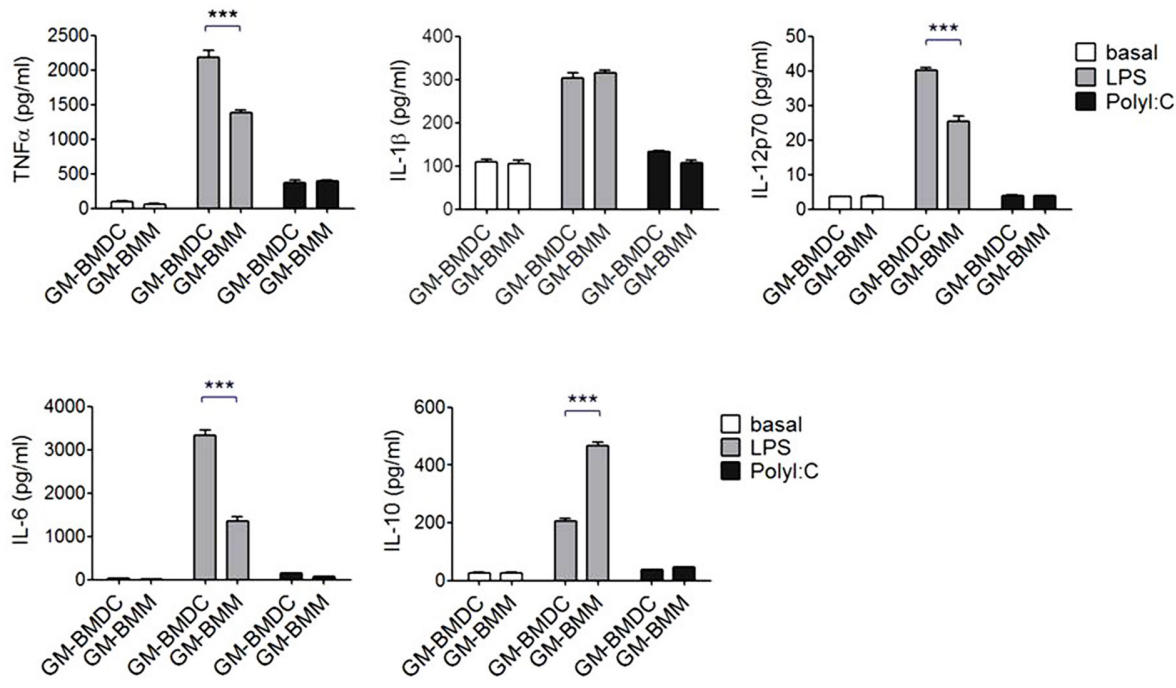

A T cell only
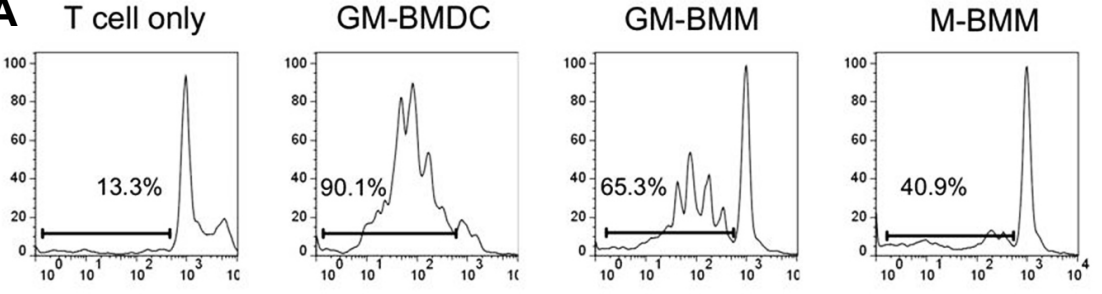

CFSE

B

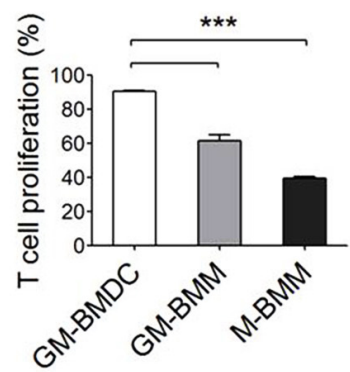

Fig. 5. Cytokine productions of GMBMDCs and GM-BMMs. Sorted GMBMDCs and GM-BMMs were seeded in 96-well plates at $5 \times 10^{5}$ cells per well. Cells were stimulated with LPS $(100 \mathrm{ng} / \mathrm{ml})$ or polyl:C (50 $\mu \mathrm{g} / \mathrm{ml}$ ) for $24 \mathrm{~h}$. TNF $\alpha$, IL-12p70, IL-6 and IL-10 in culture supernatants were quantified using ELISA. IL-1 $\beta$ was analyzed in the cell lysates using ELISA. Data are representatives of three independent experiments. Error bars, SEM, ${ }^{* *}, \mathrm{p}<$ 0.001 analyzed by one-way ANOVA with Bonferroni correction, $\mathrm{n}=3$.

Quantitative graph indicating proliferated $\mathrm{CD}^{+} \mathrm{T}$ cell percentages mixed with GM-BMDCs, GM-BMMs and M-BMMs. Error bar 0.001 analyzed by one-way ANOVA with Bonferroni correction, $n=3$.

than DCs (Crozat et al., 2010; Robbins et al., 2008). It was recently suggested that GM-CSF grown bone marrow derived macrophages are still acceptable as macrophage sources (Murray et al., 2014), however the authors worried about the contamination with DC populations. M-CSF grown macrophages, the preferred source for macrophages, show much similarity with tissue macrophages, but as we stated, GM-CSF grown macrophages have unique utility as an experimental platform to investigate inflammation specific exacerbated signaling pathways because they resemble inflammatory macrophages (Fleetwood et al., 2007). In fact, these GM-CSF grown macrophages could produce even more extensive proinflammatory cytokines compared to M-CSF grown, INF $\gamma /$ LPS polarized mac- rophages (preliminary data). Despite the remarkable inflammatory phenotype of GM-CSF grown macrophages, there is much confusion about the use of these cells because they have been used as both macrophages and DCs without any guiding criteria (Bhattacharya et al., 2011; Chung et al., 2015; Ganesh et al., 2009). Consequently, we sought to distinguish amongst the heterogenous populations of GM-CSF derived cells and especially focused on characteristics of GM-BMMs compared to GM-BMDCs in this study.

It is not surprising that we found two populations in the attached cells based on F4/80 and MHCll expression (Fig. 1A), as GM-CSF is known to produce granulocytes, macrophages and DCs from common progenitor cells (Inaba et al., 1993). We 
A
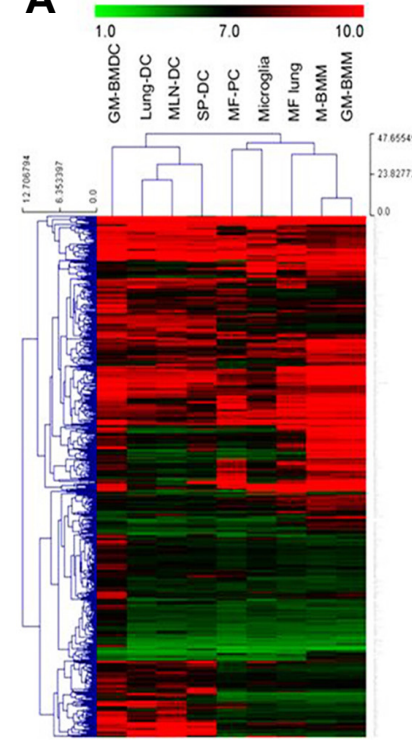

B

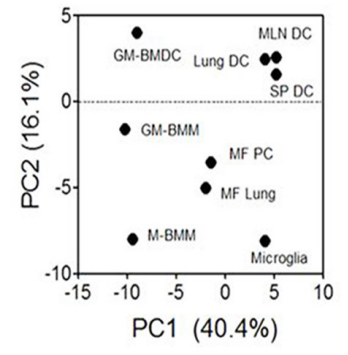

C

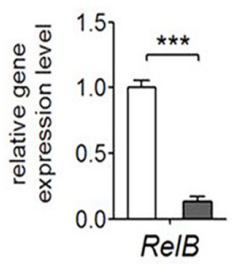

D

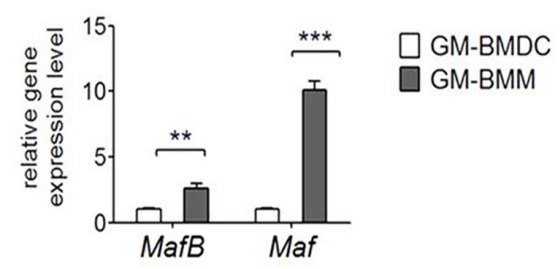

Fig. 7. Whole transcriptome analysis of GM-BMDCs and GM-BMMs. (A) Hierarchical clustering of GM-BMDCs, GM-BMMs and M-BMMs based on the $15 \%$ of genes with the greatest variability compared to populations collected by the Immunological Genome Project (GSE15907). Lung DC; $\mathrm{CD}_{103^{+}}$lung DCs, MLN DC; mesenteric lymph node CD8 ${ }^{+}$DCs, SP DC; spleen CD11b ${ }^{+}$DCs, MF PC; thioglycollate induced $\mathrm{F} 4 / 80^{\text {high }}$ macrophages, MF lung; CD11 $\mathrm{b}^{+}$lung macrophages. (B) Principal component analysis of GM-BMDCs, GM-BMMs and M-BMMs total gene expressions with

tissue resident $\mathrm{DCs}$ and macrophages used in (A). Expression data are pooled from 2 replicate microarray experiments. (C) Gene expression of RelB by GM-GMDCs and GM-BMMs was examined by real-time PCR. Error bars, SEM, ${ }^{\star \star \star} p<0.001$ analyzed by student $t$-test, $n=3$. (D) Gene expressions of MafB and Maf by GM-GMDCs and GM-BMMs were examined by real-time PCR. Error bars, SEM, ${ }^{* *} p<0.005,{ }^{\star \star \star} p<$ 0.001 analyzed by student $t$-test, $\mathrm{n}=3$.

consider the $\mathrm{MHCII}{ }^{\text {high }} \mathrm{F} 4 / 80^{\text {low }}$ population as GM-BMDCs in several aspects. They expressed high CD11c, showed a low phagocytic ability and had a standard DC-like morphology with small round shapes and many dendrites (Xu et al., 2007). In addition, they could expand $T$ cells efficiently, with similar gene ontology enrichment of antigen presentation. Recently reported classical DC-specific genes including Zbtb46, Flt3, kit and ccr7 were all upregulated in GM-BMDCs compared to GM-BMMs and M-BMMs, further indicating their resemblance with DCs (Supplementary Fig. S1), rather than macrophages. As Xu et al. (2007) had reported that DCs derived in vitro with GM-CSF/IL-4 are the equivalents of induced inflammatory Tip-DCs in vivo, we also found that GM-BMDCs extensively produced TNF $\alpha$ upon LPS stimulation in this study, indicating their inflammatory DC phenotype.

Standard $25 \mathrm{ng} / \mathrm{ml}$ of GM-CSF concentration resulted in a domination of $\mathrm{MHCII}^{\text {low }} \mathrm{F} 4 / 80^{\text {high }} \mathrm{GM}-\mathrm{BMMs}$ among attached cells. We could omit the possibility of these cells as immature DCs,(Mellman and Steinman, 2001) because they highly expressed $F 4 / 80$ on their surface and had low gene transcripts of CD80 and CD86 compared to GM-BMDCs (Supplementary Fig. S1). Macrophage specific marker CD64 (Gautier et al., 2012) was only detected on the surface of GM-BMMs, albeit was not highly expressed. A substantial level of CD11c was also detected on GM-BMMs, indicating that DCs are not the sole CD11c-positive cells. Actually the expression of F4/80 and CD11c often overlap in macrophages and DCs in nonlympoid tissues, and our results show that GM-BMMs express F4/80, $\mathrm{MHCll}$ as well as $\mathrm{CD11c}$ like with colonic macrophages and lung macrophages (Miller et al., 2012). MafB and Maf, lineage specific transcription factors for macrophages, were also expressed higher in GM-BMMs compared to GM-BMDCs (Fig. 7C). Most of all, they demonstrated remarkable phagocytic ability, comparable to M-BMMs (data not shown), and showed a morphology typical of macrophages (Fig. 4). Enriched gene ontology biological process pathways in GM-BMMs includes lysosome organization, phagocytosis, tissue remodeling, tissue homeostasis and response to wounding, all indicating macrophage specific functions of these cells (Table 1). Interestingly, the macrophage population increased in a GM-CSF concentration dependent manner, suggesting a preference toward macrophage development by newly infiltrated haematopoietic cells in a highly inflammatory environment.

In an effort to ensure the heterogeneity of GM-CSF derived cells, Helft et al. (2015) has been recently published an article in accord of most of our data. They found that CD11c CD11 $\mathrm{b}^{\text {high }}$ $\mathrm{MHCII}^{\text {Int }}$ cell population, previously considered as immature BMDCs, actually corresponds to macrophage expressing CD64, CD115 and CD14 whereas CD11 ${ }^{+} \mathrm{CD} 11 \mathrm{~b}^{\text {lnt }} \mathrm{MHCII}^{\text {hi }}$ cell population is primarily consisted with DC. Although they showed sufficient data supporting the existence of two populations in GM-CSF mouse bone marrow cultures, they originally focused on the methods of in vitro expansion of DCs thus examined on non-adherent as well as loosely adherent cells harvested by gentle washing with PBS. In this study, we aimed to focus on the characteristics of macrophages and showed contaminated DC population in attached GM-CSF derived bone marrow cells. We also revealed GM-CSF dose-dependent formation of macrophages as well as their phagocytic abilities and pronounced IL-10 productions compared to GM-BMDCs. Our data will give relevant information to readers having interests in using GM-CSF derived macrophages.

Collectively, our results clarify the characteristics of GM-CSF grown, bone marrow derived murine macrophages. They have a $\mathrm{MHCI}^{\text {low }} \mathrm{F} 4 / 80^{\text {high }} \mathrm{CD} 11 \mathrm{C}^{+} \mathrm{CD} 64^{+}$phenotype and are efficient at phagocytosis. GM-CSF also simultaneously produces a MHCII high $F 4 / 80^{\text {low }}$ attached DC population, but this rate of formation diminishes with increasing GM-CSF concen- 
tration. Using mixed populations in experimental settings is common; however, it may be desirable to enhance macrophage purity as much as possible. This study might give useful information for researchers using bone marrow derived macrophages.

Note: Supplementary information is available on the Molecules and Cells website (www.molcells.org).

\section{ACKNOWLEDGEMENTS}

This work was supported by Bumsuk Academic Research Fund in 2014.

\section{REFERENCES}

Banchereau, J., and Steinman, R.M. (1998). Dendritic cells and the control of immunity. Nature 392, 245-252.

Becker, L., Liu, N.C., Averill, M.M., Yuan, W., Pamir, N., Peng, Y., Irwin, A.D., Fu, X., Bornfeldt, K.E., and Heinecke, J.W. (2012). Unique proteomic signatures distinguish macrophages and dendritic cells. PLoS One 7, e33297.

Bhattacharya, P., Gopisetty, A., Ganesh, B.B., Sheng, J.R., and Prabhakar, B.S. (2011). GM-CSF-induced, bone-marrow-derived dendritic cells can expand natural Tregs and induce adaptive Tregs by different mechanisms. J. Leukocyte Biol. 89, 235-249.

Cebon, J., Layton, J.E., Maher, D., and Morstyn, G. (1994). Endogenous haemopoietic growth factors in neutropenia and infection. Br. J. Haematol. 86, 265-274.

Cheers, C., Haigh, A.M., Kelso, A., Metcalf, D., Stanley, E.R., and Young, A.M. (1988). Production of colony-stimulating factors (CSFs) during infection: separate determinations of macrophagegranulocyte-, granulocyte-macrophage-, and multi-CSFs. Infect. Immun. 56, 247-251.

Chung, S., Ranjan, R., Lee, Y.G., Park, G.Y., Karpurapu, M., Deng, J., Xiao, L., Kim, J.Y., Unterman, T.G., and Christman, J.W. (2015). Distinct role of FoxO1 in M-CSF- and GM-CSFdifferentiated macrophages contributes LPS-mediated IL-10: implication in hyperglycemia. J. Leukocyte Biol. 97, 327-339.

Crozat, K., Guiton, R., Guilliams, M., Henri, S., Baranek, T., Schwartz-Cornil, I., Malissen, B., and Dalod, M. (2010). Comparative genomics as a tool to reveal functional equivalences between human and mouse dendritic cell subsets. Immunol. Rev. 234, 177-198.

Egawa, M., Mukai, K., Yoshikawa, S., Iki, M., Mukaida, N., Kawano, Y., Minegishi, Y., and Karasuyama, H. (2013). Inflammatory monocytes recruited to allergic skin acquire an anti-inflammatory M2 phenotype via basophil-derived interleukin-4. Immunity 38, 570-580.

Fleetwood, A.J., Lawrence, T., Hamilton, J.A., and Cook, A.D. (2007). Granulocyte-macrophage colony-stimulating factor (CSF) and macrophage CSF-dependent macrophage phenotypes display differences in cytokine profiles and transcription factor activities: implications for CSF blockade in inflammation. J. Immunol. 178, 5245-5252.

Ganesh, B.B., Cheatem, D.M., Sheng, J.R., Vasu, C., and Prabhakar, B.S. (2009). GM-CSF-induced CD11C+CD8a-dendritic cells facilitate Foxp3+ and IL-10+ regulatory $\mathrm{T}$ cell expansion resulting in suppression of autoimmune thyroiditis. Int. Immunol. 21, 269-282.

Gautier, E.L., Shay, T., Miller, J., Greter, M., Jakubzick, C., Ivanov, S., Helft, J., Chow, A., Elpek, K.G., Gordonov, S., et al. (2012). Gene-expression profiles and transcriptional regulatory pathways that underlie the identity and diversity of mouse tissue macrophages. Nat. Immunol. 13, 1118-1128.

Hashimoto, D., Miller, J., and Merad, M. (2011). Dendritic cell and macrophage heterogeneity in vivo. Immunity 35, 323-335.

Helft, J., Böttcher, J., Chakravarty, P., Zelenay, S., Huotari, J., Schraml, B.U., Goubau, D., and Reis e Sousa, C. (2015). GMCSF mouse bone marrow cultures comprise a heterogeneous population of $\mathrm{CD} 11 \mathrm{c}(+) \mathrm{MHCll}(+)$ macrophages and dendritic cells. Immunity 42, 1197-1211.

Heng, T.S., Painter, M.W., and Immunological Genome Project, C. (2008). The Immunological Genome Project: networks of gene expression in immune cells. Nat. Immunol. 9, 1091-1094.

Hercus, T.R., Thomas, D., Guthridge, M.A., Ekert, P.G., King-Scott, J., Parker, M.W., and Lopez, A.F. (2009). The granulocytemacrophage colony-stimulating factor receptor: linking its structure to cell signaling and its role in disease. Blood 114, 1289-1298.

Inaba, K., Inaba, M., Deguchi, M., Hagi, K., Yasumizu, R., Ikehara, S., Muramatsu, S., and Steinman, R.M. (1993). Granulocytes, macrophages, and dendritic cells arise from a common major histocompatibility complex class II-negative progenitor in mouse bone marrow. Proc. Natl. Acad. Sci. USA 90, 3038-3042.

Mellman, I., and Steinman, R.M. (2001). Dendritic cells: specialized and regulated antigen processing machines. Cell 106, 255-258.

Miller, J.C., Brown, B.D., Shay, T., Gautier, E.L., Jojic, V., Cohain, A., Pandey, G., Leboeuf, M., Elpek, K.G., Helft, J., et al. (2012). Deciphering the transcriptional network of the dendritic cell lineage. Nat. Immunol. 13, 888-899.

Murray, P.J., Allen, J.E., Biswas, S.K., Fisher, E.A., Gilroy, D.W., Goerdt, S., Gordon, S., Hamilton, J.A., Ivashkiv, L.B., Lawrence, T., et al. (2014). Macrophage activation and polarization: nomenclature and experimental guidelines. Immunity 41, 14-20.

Nikolic, T., de Bruijn, M.F., Lutz, M.B., and Leenen, P.J. (2003). Developmental stages of myeloid dendritic cells in mouse bone marrow. Int. Immunol. 15, 515-524.

Paine, R., 3rd, Morris, S.B., Jin, H., Wilcoxen, S.E., Phare, S.M., Moore, B.B., Coffey, M.J., and Toews, G.B. (2001). Impaired functional activity of alveolar macrophages from GM-CSFdeficient mice. Am. J. Physiol. Lung Cell. Mol. Physiol. 281, L1210-1218.

Robbins, S.H., Walzer, T., Dembele, D., Thibault, C., Defays, A., Bessou, G., Xu, H., Vivier, E., Sellars, M., Pierre, P., et al. (2008). Novel insights into the relationships between dendritic cell subsets in human and mouse revealed by genome-wide expression profiling. Genome Biol. 9, R17.

Saraiva, M., and O'Garra, A. (2010). The regulation of IL-10 production by immune cells. Nat. Rev. Immunol. 10, 170-181.

Satpathy, A.T., Wu, X., Albring, J.C., and Murphy, K.M. (2012). $\mathrm{Re}(\mathrm{de})$ fining the dendritic cell lineage. Nat. Immunol. 13, 11451154.

Seok, S.H., Heo, J.I., Hwang, J.H., Na, Y.R., Yun, J.H., Lee, E.H. Park, J.W., and Cho, C.H. (2013). Angiopoietin-1 elicits proinflammatory responses in monocytes and differentiating macrophages. Mol. Cells 35, 550-556.

Xu, Y., Zhan, Y., Lew, A.M., Naik, S.H., and Kershaw, M.H. (2007). Differential development of murine dendritic cells by GM-CSF versus Flt3 ligand has implications for inflammation and trafficking. J. Immunol. 179, 7577-7584.

Zhang, Y., Harada, A., Wang, J.B., Zhang, Y.Y., Hashimoto, S. Naito, M., and Matsushima, K. (1998). Bifurcated dendritic cell differentiation in vitro from murine lineage phenotype-negative ckit+ bone marrow hematopoietic progenitor cells. Blood 92, 118128. 